PROCEEDINGS OF THE

AMERICAN MATHEMATICAL SOCIETY

Volume 133, Number 9, Pages 2491-2494

S 0002-9939(05)07564-7

Article electronically published on April 12, 2005

\title{
QUASI-HYPERBOLIC PLANES IN HYPERBOLIC GROUPS
}

\author{
MARIO BONK AND BRUCE KLEINER
}

(Communicated by Ronald A. Fintushel)

\begin{abstract}
The hyperbolic plane $\mathbb{H}^{2}$ admits a quasi-isometric embedding into every hyperbolic group which is not virtually free.
\end{abstract}

The purpose of this note is to prove the following theorem which answers a question posed by P. Papasoglu:

Theorem 1. The hyperbolic plane $\mathbb{H}^{2}$ admits a quasi-isometric embedding into a hyperbolic group if and only if the group is not virtually free.

A map $f: X \rightarrow Y$ between two metric spaces $\left(X, d_{X}\right)$ and $\left(Y, d_{Y}\right)$ is called a quasi-isometric embedding if there exist constants $\lambda \geq 1$ and $K \geq 0$ such that

$$
\frac{1}{\lambda} d_{X}(x, y)-K \leq d_{Y}(f(x), f(y)) \leq \lambda d_{X}(x, y)+K
$$

for all $x, y \in X$. A group is virtually free if it contains a free subgroup of finite index. We refer to [9] for the definition of hyperbolic groups and related concepts from the theory of Gromov hyperbolic spaces. Every Gromov hyperbolic space $X$ has a boundary $\partial_{\infty} X$ which carries a class of canonical visual metrics. These metrics are bi-Lipschitz equivalent to distance functions of the form

$$
d_{w, \epsilon}(a, b)=\exp \left(-\epsilon(a, b)_{w}\right), \quad a, b \in \partial_{\infty} X
$$

where $w \in X$ is a base point, $\epsilon>0$ is sufficiently small, and $(a, b)_{w}$ denotes the Gromov product of the points $a$ and $b$ with respect to $w$ (cf. [9, Ch. 7]).

Corollary 2. The boundary of a hyperbolic group (equipped with any visual metric) contains a quasi-circle if and only if the group is not virtually free.

By definition a quasi-circle is a metric circle which admits a quasisymmetric parametrization by the unit circle $\mathbb{S}^{1} \subset \mathbb{R}^{2}$ (see [10] for the definition and basic facts about quasisymmetric maps). Since the boundary of a virtually free group is totally disconnected, the "only if" part of the corollary is obvious.

One of the main ingredients in the proof of the theorem is a result by Tukia [14 which insures the existence of quasi-arcs with given endpoints inside certain subsets of $\mathbb{R}^{n}$ (a quasi-arc is a quasisymmetric image of the interval $[0,1]$ ). The authors thank Juha Heinonen for drawing their attention to Tukia's paper, which allowed them to substantially shorten the proof of the next proposition.

Received by the editors January 21, 2003.

2000 Mathematics Subject Classification. Primary 20F67.

The first author was supported by NSF grant DMS-0200566.

The second author was supported by NSF grants DMS-9972047 and DMS-0204506. 
To state this proposition, we need two more definitions. A metric space $Z$ is doubling if there exists a constant $N \in \mathbb{N}$ with the following property: If $B$ is an arbitrary ball in $Z$ and $R$ is its radius, then $B$ can be covered by $N$ balls of radius $R / 2$. The metric space $Z$ is linearly connected if there exists a constant $L$ such that for all $x, y \in Z$ there is a connected subset $S \subset Z$ of diameter at most $\operatorname{Ld}(x, y)$ containing $\{x, y\}$.

Proposition 3. If $X$ is a complete, doubling, and linearly connected metric space, then any two distinct points in $X$ are the endpoints of a quasi-arc.

Proof. Let $d$ denote the metric on $X$, and pick $\alpha \in(0,1)$. Since $X$ is doubling, there exists $n \in \mathbb{N}$ such that the " $\alpha$-snowflaked" metric space $\left(X, d^{\alpha}\right)$ can be embedded into $\mathbb{R}^{n}$ (equipped with the usual metric) by a bi-Lipschitz mapping (this follows from Assouad's Embedding Theorem [1, 2.6. Prop.]; see [10, Thm. 12.2] for the version of this theorem used here). Let $Z$ denote the image of such an embedding. Then $Z$ is complete and linearly connected, since $X$ has these properties. Hence any two distinct points in $Z$ are the endpoints of a quasi-arc in $Z$ (this is [14, Thm $1 \mathrm{~A}]$ expressed in our terminology; see the introduction of 14 for a discussion). Since quasi-arcs in $Z$ pull back to quasi-arcs in $X$, the result follows.

Proposition 4. If $G$ is a 1-ended hyperbolic group, then $\partial_{\infty} G$ equipped with any visual metric d is compact, doubling, connected, and linearly connected.

Proof. It is easy to show that $\partial_{\infty} G$ is compact [9. p. 123, 9. Prop.] and doubling [4, Sect. 9]. Since the group $G$ is 1 -ended, its boundary $\partial_{\infty} G$ is connected.

It remains to prove linear connectedness (note that this is a stronger quantitative version of local connectedness which was established in this context in 2, Prop. $3.3]$ ). Given two points $x$ and $y$ in a metric space $(Z, d)$, and $\lambda>0$, a $\lambda$-chain from $x$ to $y$ is a sequence of points $x=z_{1}, \ldots, z_{k}=y$ such that $d\left(z_{i}, z_{i+1}\right) \leq \lambda$ for all $1 \leq i<k$. The length of a $\lambda$-chain is the number of points in the chain.

Lemma 5. There is a number $N \in \mathbb{N}$ such that for all $x, y \in \partial_{\infty} G$ there is a $\frac{1}{2} d(x, y)$-chain of length at most $N$ from $x$ to $y$.

Proof. If not, there are sequences $\left\{x_{k}\right\}$ and $\left\{y_{k}\right\}$ in $\partial_{\infty} G$ such that the shortest $\frac{1}{2} d\left(x_{j}, y_{j}\right)$-chain from $x_{j}$ to $y_{j}$ has length $j$. The boundary $\partial_{\infty} G$ is compact and connected, so clearly $r_{j}:=d\left(x_{j}, y_{j}\right) \rightarrow 0$ as $j \rightarrow \infty$. In view of the doubling property, the sequence $\left(\partial_{\infty} G, \frac{1}{r_{j}} d, x_{j}\right)$ of pointed metric spaces subconverges to a limit $\left(W, d_{W}, x_{\infty}\right)$ with respect to pointed Gromov-Hausdorff convergence [7] Thm. 8.1.10]. We can then find a point $y_{\infty} \in W$ such that $d_{W}\left(x_{\infty}, y_{\infty}\right)=1$ and there is no $\lambda$-chain from $x_{\infty}$ to $y_{\infty}$ for any $\lambda<\frac{1}{2}$. This implies that $W$ is not connected. By [3 Lemma 5.2], the limit space $W$ is homeomorphic to $\partial_{\infty} G \backslash\{z\}$ for some $z \in \partial_{\infty} G$, and so $z$ is a "global cut point" of $\partial_{\infty} G$.

On the other hand, it is a well-known (and deep) fact if $\partial_{\infty} G$ is connected, then $\partial_{\infty} G$ has no global cut points (see [13], 6, Thm. 9.3], [5. Cor. 0.3]). This is a contradiction.

Now suppose $x$ and $y$ are arbitrary points in $\partial_{\infty} G$. By the lemma we can find a $\frac{1}{2} d(x, y)$-chain $S_{1}=\left\{z_{1}, \ldots, z_{k}\right\}$ which joins $x$ to $y$ and has length $k \leq N$. Now define $S_{2}$ by adding, for each $1 \leq i<k$, the points in a $\frac{1}{2} d\left(z_{i}, z_{i+1}\right)$-chain joining $z_{i}$ to $z_{i+1}$. Repeating this process inductively, we obtain a nested sequence of sets $S_{1} \subset \ldots \subset S_{j} \subset \ldots$ The closure $S$ of the union $\bigcup_{j} S_{j}$ will be a connected set 
containing $x$ and $y$ whose diameter does not exceed $L d(x, y)$, where $L$ is a constant independent of $x$ and $y$. This shows that $\partial_{\infty} G$ is linearly connected.

The proofs of Theorem 1 and Corollary 2. We first assume that $G$ a hyperbolic group which is not virtually free, and prove that there is a quasi-isometric embeddding $\mathbb{H}^{2} \rightarrow G$ and a quasi-circle in $\partial_{\infty} G$. Every hyperbolic group is finitely presentable 9 , p. 76, 17. Prop.]. Hence there is a finite graph of groups decomposition of $G$ where all edge groups are finite, and all vertex groups have at most one end [ $\underline{8}$, Theorem 6.2.14]. Since $G$ is not virtually free, one of the vertex groups $G_{0}$ is 1-ended [8, Theorem 6.2.12]. The group $G_{0}$ is quasi-isometrically embedded in $G$, since this is true for every vertex group in a graph of groups decomposition with finite edge groups [11, Rem. 3.6]. This implies that $G_{0}$ is also a hyperbolic group. So without loss of generality we may assume that $G$ itself is 1-ended.

Let $\partial_{\infty} G$ denote the boundary of $G$ equipped with a visual metric. By Proposition 4, the hypotheses of Proposition 3 are satisfied for $\partial_{\infty} G$. Hence there is a quasisymmetric map $[0,1] \rightarrow \partial_{\infty} G$. Since $[0,1]$ is quasisymmetrically homeomorphic to the boundary of a hyperbolic half-plane $\mathbb{H}_{+}^{2} \subset \mathbb{H}^{2}$, we conclude that there is a quasi-isometric embedding $\mathbb{H}_{+}^{2} \rightarrow G$ (see the proof of Prop. 4.2 in 12, for example). In particular, one can quasi-isometrically embed arbitrarily large balls $B \subset \mathbb{H}^{2}$ into $G$ with uniform constants for the quasi-isometric embeddings. By precomposing with isometries in $\mathbb{H}^{2}$, post-composing with left translations in the group $G$, and applying a compactness argument based on the Arzelà-Ascoli Theorem, we can obtain a quasi-isometric embedding $\mathbb{H}^{2} \rightarrow G$ as a limit. A quasi-isometric embedding of a Gromov hyperbolic space $X$ into a Gromov hyperbolic space $Y$ induces a quasisymmetric embedding of $\partial_{\infty} X$ into $\partial_{\infty} Y$ (see [4, Thm. 6.5], where this is essentially proved); since $\partial_{\infty} \mathbb{H}^{2}$ is quasisymmetrically equivalent to $\mathbb{S}^{1}$, we deduce that the boundary $\partial_{\infty} G$ contains a quasi-circle.

Now suppose $G$ is virtually free. It follows that $\partial_{\infty} G$ is totally disconnected, and therefore cannot contain a quasi-circle. This then implies that there is no quasi-isometric embedding $\mathbb{H}^{2} \rightarrow G$.

This completes the proofs of the theorem and corollary.

Remarks. There are various open questions that are related to our theorem. For example, Papasoglu has asked if every one-ended finitely presented group $G$ contains a quasi-plane - the image of a uniform embedding $P \rightarrow G$, where $P$ is a complete Riemannian plane of bounded geometry. A problem due to Gromov is whether every 1-ended hyperbolic group $G$ is the target of a homomorphism $\phi: S \rightarrow G$, where $S$ is a surface group and $\phi$ does not factor through a free group.

\section{REFERENCES}

1. P. Assouad, Plongements lipschitziens dans $\mathbf{R}^{n}$, Bull. Soc. Math. France 111 (1983), 429-448. MR0763553 (86f:54050)

2. M. Bestvina and G. Mess, The boundary of negatively curved groups, J. Amer. Math. Soc. 4 (1991), 469-481. MR.1096169 (93j:20076)

3. M. Bonk and B. Kleiner, Rigidity for quasi-Möbius group actions, J. Differential Geom. 61 (2002), no. 1, 81-106. MR.1949785 (2004b:53059)

4. M. Bonk and O. Schramm, Embeddings of Gromov hyperbolic spaces, Geom. Funct. Anal. 10 (2000), 266-306. MR1771428 (2001g:53077)

5. B. H. Bowditch, Connectedness properties of limit sets, Trans. Amer. Math. Soc. 351 (1999), 3673-3686. MR1624089|(2000d:20056) 
6. B.H. Bowditch, Boundaries of strongly accessible hyperbolic groups, The Epstein birthday schrift, Geom. Topol. Monogr., vol. 1, Geom. Topol. Publ., Coventry, 1998, pp. 51-97 (electronic). MR1668331 (2000d:20059)

7. D. Burago, Y. Burago, and S. Ivanov, A course in metric geometry, Graduate Studies in Mathematics, vol. 33, American Mathematical Society, Providence, RI, 2001. MR1835418 (2002e:53053)

8. D.J. Collins and H. Zieschang, Combinatorial group theory and fundamental groups, Algebra VII. Combinatorial group theory. Applications to Geometry, Encycl. Math. Sci. 58, Springer, Berlin, 1993, pp. 1-166. MF,1265270

9. É. Ghys and P. de la Harpe (eds.), Sur les groupes hyperboliques d'après Mikhael Gromov, Birkhäuser, Boston, MA, 1990. MR,1086648 (92f:53050)

10. J. Heinonen, Lectures on analysis on metric spaces, Universitext, Springer-Verlag, New York, 2001. MR 1800917 (2002c:30028)

11. I. Kapovich, Quasiconvexity and amalgams, Internat. J. Algebra Comput. 7 (1997), 771-811. MR 1482968 (98k:20057)

12. F. Paulin, Un groupe hyperbolique est déterminé par son bord, J. London Math. Soc. (2) 54 (1996), no. 1, 50-74. MR1395067 (97d:20042)

13. G.A. Swarup, On the cut point conjecture, Electron. Res. Announc. Amer. Math. Soc. 2 (1996), 98-100 (electronic). MR1412948 (97f:20048)

14. P. Tukia, Spaces and arcs of bounded turning, Michigan Math. J. 43 (1996), 559-584. MR $1420592(98 \mathrm{a}: 30028)$

Department of Mathematics, University of Michigan, Ann Arbor, Michigan 481091109

E-mail address: mbonk@umich.edu

Department of Mathematics, University of Michigan, Ann Arbor, Michigan 481091109

E-mail address: bkleiner@umich.edu 